OPEN ACCESS

Edited by:

Marcin Okrój,

Intercollegiate Faculty of

Biotechnology of University of Gdańsk and Medical University

of Gdańsk, Poland

Reviewed by:

Frank J. Beurskens,

Genmab, Netherlands

Daniel Ajona,

University of Navarra, Spain

*Correspondence:

Regina Michelis

ReginaM@gmc.gov.il

${ }^{+}$These authors have contributed equally to this work

Specialty section:

This article was submitted to

Molecular Innate Immunity,

a section of the journal

Frontiers in Immunology

Received: 07 September 2020 Accepted: 31 December 2020

Published: 11 February 2021

Citation:

Naseraldeen N, Michelis R, Barhoum M, Chezar J, Tadmor T,

Aviv A, Shvidel L, Litmanovich A, Shehadeh M, Stemer G, Shaoul E and Braester A (2021) The Role of Alpha 2 Macroglobulin in IgG-Aggregation and Chronic Activation of the Complement

System in Patients With Chronic

Lymphocytic Leukemia.

Front. Immunol. 11:603569. doi: 10.3389/fimmu.2020.603569

\section{The Role of Alpha 2 Macroglobulin in IgG-Aggregation and Chronic Activation of the Complement System in Patients With Chronic Lymphocytic Leukemia}

\author{
Naseba Naseraldeen ${ }^{1,2 t}$, Regina Michelis ${ }^{1 * t}$, Masad Barhoum ${ }^{2,3}$, Judith Chezar ${ }^{3}$, \\ Tamar Tadmor ${ }^{4,5}$, Ariel Aviv ${ }^{6}$, Lev Shvidel ${ }^{7,8}$, Adi Litmanovich ${ }^{2}$, Mona Shehadeh ${ }^{9}$, \\ Galia Stemer $^{6}$, Ety Shaoul ${ }^{3}$ and Andrei Braester ${ }^{2,3}$ \\ 1 The Institute for Medical Research, Galilee Medical Center, Nahariya, Israel, ${ }^{2}$ Azrieli Faculty of Medicine, Bar Ilan University, \\ Safed, Israel, ${ }^{3}$ Institute of Hematology, Galilee Medical Center, Nahariya, Israel, ${ }^{4}$ Hematology Unit, Bnai Zion Medical Center, \\ Haifa, Israel, 5 The Ruth and Bruce Rappaport Faculty of Medicine, Technion, Haifa, Israel, ${ }^{6}$ Department of Hematology, \\ Emek Medical Center, Afula, Israel, 7 Hematology Institute, Kaplan Medical Center, Rehovot, Israel, ${ }^{8}$ Faculty of Medicine, \\ Hebrew University, Jerusalem, Israel, 9 Biochemistry Laboratory, Galilee Medical Center, Nahariya, Israel
}

Chronic lymphocytic leukemia (CLL) is the most common leukemia in adults in the western world. One of the treatments offered for CLL is immunotherapy. These treatments activate various cellular and biochemical mechanisms, using the complement system. Recently it was shown that the complement system in CLL patients is persistently activated at a low level through the classical pathway (CP). The mechanism of chronic CP activation involves the formation of IgG-hexamers (IgG-aggregates). According to recent studies, formation of ordered IgG-hexamers occurs on cell surfaces via specific interactions between Fc regions of the $\operatorname{lgG}$ monomers, which occur after antigen binding. The present study investigated the formation of IgG-hexamers in CLL patients and normal (non-malignant) controls (NC), their ability to activate complement, their incidence as cell-free and cellbound forms and the identity of the antigen causing their formation. Sera from 30 patients and 12 NC were used for separation of IgG- aggregates. The obtained IgG- aggregates were measured and used for assessment of $\mathrm{CP}$ activation. For evaluation of the presence of IgG- aggregates on blood cells, whole blood samples were stained and assessed by flow cytometry. Serum levels of lgG- aggregates were higher in CLL and they activated the complement system to a higher extent than in NC. Alpha 2 macroglobulin (A2M) was identified as the antigen causing the hexamerization/aggregation of $\operatorname{lgG}$, and was found to be part of the hexamer structure by mass spectrometry, Western blot and flow cytometry analysis. The presence of A2M-IgG-hexamers on B-cells suggests that it may be formed on B cells surface and then be detached to become cell-free. Alternatively, it may form in the plasma and then attach to the cell surface. The exact time course of A2M-lgG- 
hexamers formation in CLL should be further studied. The results in this study may be useful for improvement of current immunotherapy regimens.

Keywords: chronic lymphocytic leukemia, classical pathway, complement system, IgG-hexamers, alpha 2 macroglobulin

\section{INTRODUCTION}

Chronic lymphocytic leukemia (CLL) is the most common leukemia in adults in the western world. It affects the B-type lymphocytes in the bone marrow in $95 \%$ of the patients, and is characterized by increased numbers of monoclonal Blymphocytes $\left(>5 \times 10^{3} / \mu \mathrm{l}\right)$ that express specific antigens $(\mathrm{CD} 5$, CD19, CD20, CD23) on their surface (1).

One of the treatments offered for CLL patients is immunotherapy which allows the immune system to identify cancer cells or train the immune system to fight the malignant $\mathrm{B}$ cells (2). The mechanisms involved by the immunotherapeutic monoclonal antibodies are complement dependent cytotoxicity (CDC), antibody dependent cell-mediated cytotoxicity (ADCC), lysosomal membrane permeability (LMP) (3) and phagocytosis $(4,5)$. The complement system is an ancient defense mechanism preceding adaptive immunity (6). Three complement pathways utilize the nine central proteins of this system (C1-C9): the classical (CP), alternative (AP) and lectin pathways. The interaction of the first component in each pathway with an activator leads to an ordered cascade activation, typical for each pathway (7). All the complement pathways eventually produce the Membrane Attack Complex (MAC, C5b-9), a cytolytic end product, which causes osmotic lysis of the pathogen/target cell (8). The complement system in CLL patients shows decreased levels of complement components (9), decreased CP activity and chronic activation at a low level $(10,11)$. The $\mathrm{CP}$ is involved in this chronic activity, and the decrease in $\mathrm{CP}$ activity was assumed to be due to fatigue (10).

The $\mathrm{CP}$ is initiated by the binding of $\mathrm{Clq}$ to the $\mathrm{Fc}$ regions of antigen-bound immunoglobulins type G or M (IgG or IgM) (7). Conformational changes in $\mathrm{Clq}$ lead to the activation of $\mathrm{C} 1 \mathrm{r}$ which, in turn, activates $\mathrm{C} 1 \mathrm{~s}(6,12)$. All these proteins are serine proteases that combine $\mathrm{C} 1$, the first component of the $\mathrm{CP}$ (13). Clq shows weak binding to the $\mathrm{Fc}$ regions of monomeric, nonaggregated IgG, while in the presence of aggregated/hexamerized IgG, that occurs after antigen binding to the Fab domain, the strength of the binding increases and the $\mathrm{CP}$ is activated efficiently $(14,15)$. The formation of the IgG-hexamers is not entirely understood. According to some studies the binding of the antigen on cells causes specific non-covalent interactions between $\mathrm{Fc}$ segments of IgG which lead to the arrangement of hexamers (16), suggesting that IgG can form ordered hexamers only on cell surfaces (15). Recently we have demonstrated the presence of aggregates in plasma of CLL patients, which are not bound to cell surfaces (cell-free) (17). The antigen stimulating the formation of these IgG-hexamers may have great importance for the chronic CP activation in CLL, and is described in this study for the first time.

\section{MATERIALS AND METHODS}

\section{Subjects}

Blood samples were collected from 30 naïve CLL patients and 12 normal (non-malignant) controls (NC). Plasma and sera were separated and frozen at $-80^{\circ} \mathrm{C}$. The rest of the samples were analyzed in the biochemistry and hematology laboratories. Samples were carefully collected and handled as described (18) in order to avoid spontaneous complement activation. The study was approved by the Helsinki Committee (Institutional Review Board) of Galilee Medical Center, Nahariya, Israel. Patients were divided according to the detection of Ig-C5a (by Western blot analysis), a marker of chronic complement activity $(10,18)$.

\section{Separation of IgG-hexamers}

Affinity Purification of Total IgG

Cell-free IgG were separated from sera/plasma using a commercial kit for total IgG extraction, based on affinity chromatography, according to the manufacturer's instructions (Protein G HP SpinTrap ${ }^{\mathrm{TM}}$, GE Healthcare). The final stage of IgG separation included elution by a low $\mathrm{pH}(0.1 \mathrm{M}$ glycine- $\mathrm{HCl}$, $\mathrm{pH}$ 2.7), followed immediately by $\mathrm{pH}$ neutralization, according to the manufacturer's instructions. The IgG separated by this kit includes all IgG molecules, i.e. monomers and hexamers. According to the manufacturer, other immunoglobulins (IgM etc.) are not separated by the kit. Fractions of non-IgG proteins produced during the early stages of the purification procedure, were stored at $4^{\circ} \mathrm{C}$ for later use. Some of the IgG-hexamer separations were repeated using a different method, the Melon Gel IgG Purification Kit (Thermo Fisher Scientific), which does not include any exposure of the samples to acidic conditions.

\section{Size Selection}

The total IgG fraction separated using the protein $G$ kit was transferred to a filtration column with a cutoff of $1000 \mathrm{kDa}$ (Vivaspin, Sartorius), and centrifuged for $3 \mathrm{~min}$ at $4 \mathrm{~K} \mathrm{~g}$ at $4^{\circ} \mathrm{C}$. Due to the high molecular weight of IgG-hexamers, $>1000 \mathrm{kDa}$, they are retained on top of the column while monomeric $\operatorname{IgG}$ move to the bottom. Protein concentrations of the samples remaining on top and at the bottom of the filtration column were measured by Nanodrop (Thermo scientific), and were used, with the sample volumes, for calculation of total protein amount. Monomeric IgG were also stored at $4^{\circ} \mathrm{C}$ for later use.

\section{Protein Staining Methods Silver Stain}

To visualize the extracted IgG-hexamers, samples obtained after the filtration step were separated by SDS-PAGE and stained using a silver stain kit, (ProteoSilver ${ }^{\mathrm{TM}}$ Plus Silver Stain Kit- 
SIGMA). Results were documented using a gel imaging system (G-BOX, Syngene). To assure that the amount of the silverstained proteins is sufficient for sequencing/mass spectrometry, the gels were de-stained and stained again with Coomassie blue (CB).

\section{Western Blot Analysis}

Sera/plasma of CLL patients were tested to identify specific proteins related to the complement system (the Ig-C5a complex, C5, IgG, $\alpha-2$ macroglobulin $[A 2 M])$. Proteins were separated by SDS-PAGE with or without denaturation, transferred to a nitrocellulose membrane, and the studied protein was identified with the appropriate primary [anti human IgG (Sigma), anti human C5 (Quidel), anti human A2M (Gentex)], and the appropriate secondary antibodies. Signals were developed using an ECL kit (Immobilon ${ }^{\circledR}$ Forte, Millipore) and documented using a gel imaging system (G-BOX, Syngene).

\section{Complement Activity}

\section{Complement Activity Assay}

In order to assess CP activity, IgG-hexamers or in-vitro aggregated IgG (19), were incubated with diluted (1:20) normal sera as described (10). The IgG-hexamers obtained from $10 \mu \mathrm{l}$ serum were used for the assay. Non-IgG and monomeric-IgG, obtained during the IgG-hexamers separation procedure, were acetone precipitated (due to their high volume) and incubated with diluted normal serum, C1q depleted serum (Quidel) or Factor B depleted serum (Quidel). The activity was measured by the levels of MAC/sC5b-9, quantified using ELISA.

\section{ELISA}

To quantify the complement activation product MAC, an ELISA kit (sC5b-9 PLUS EIA, Quidel) was used according to the manufacturer's instructions. The results were measured using an ELISA reader (CARIOSKAN LUX, Thermo scientific).

\section{Cell Analysis}

\section{Flow Cytometry}

Whole blood samples were diluted to $10^{4} / \mu$ l white blood cells (WBC), washed with PBS three times, and stained with fluorescent anti-CD19-PC7 (Beckman), anti CD45-APC (Beckman), anti-C1FITC (Assaypro), anti A2M-PE (Assaypro), anti CD91-Per-CPeF710 (Invitrogen, eBioscience), A2M-PerCP (Assaypro) and antiGRP78 (anti-Bip, Cell Signaling Technology) antibodies. AntiCD45 stains all the WBC while anti-CD19 marks B lymphocytes. $\mathrm{C} 1$ binds IgG only in its hexameric form and thus anti-C1 antibodies can detect cells to which IgG-hexamers and C1 are bound. Anti-CD91 and anti-78 kDa Glucose-Regulated Protein (GRP78) detect these A2M receptors on lymphocytes $(20,21)$. After incubation with the antibodies, the red blood cells were lysed with VersaLyse (Beckman). All incubations were performed at room temperature, in the dark, for $10 \mathrm{~min}$. Cell staining was assessed by a Flow cytometer (NAVIOS, Beckman coulter).

\section{Mass Spectrometry (Protein Sequencing)}

The IgG-hexamers prepared from patients' sera were separated by SDS-PAGE and silver stained (with ProteoSilver ${ }^{\mathrm{TM}}$ plus,
Sigma). The heavy $(\gamma)$ and light chains were identified by their molecular mass and high abundance. All other protein bands were excised from the gels and subjected to mass spectrometry at the Smoler Protein Research Center (Technion - Israel Institute of Technology, Haifa, Israel).

\section{Monomeric IgG-Aggregation with A2M}

The samples used for these experiments were the monomeric IgG fractions, obtained after IgG affinity columns and size selection (i.e. the sample at the bottom of the $1000 \mathrm{kDa}$ Vivaspin column). Monomeric IgG concentrations were measured by Nanodrop and $7.5 \mu \mathrm{g}$ from each sample were incubated with $15.8 \mu \mathrm{g}$ of $\mathrm{A} 2 \mathrm{M}$. These protein amounts give a molar ratio of 1:1 for the IgG vs. the $\mathrm{A} 2 \mathrm{M}$ dimer, namely one monomeric IgG molecule to one A2M dimer. As a control, monomeric IgG was incubated with human serum albumin (HSA) at a molar ratio of 1:1, or without any protein. All incubations were performed in $100 \mu \mathrm{l}$ PBS for $2 \mathrm{hr}$ at room temperature in glass test tubes (as A2M adsorps to non-glass surfaces) (22). After the incubation, samples were used for size selection using the $1,000 \mathrm{kDa}$ Vivaspin columns (Sartorius). The volumes of the samples obtained on top and bottom of the columns were measured, and the IgG concentrations were quantified using a human-IgG ELISA kit (Invitrogen).

\section{Statistical Analysis}

Data were analyzed by Kolmogorov-Smirnov, Kruskal-Wallis and by Mann-Whitney tests, as appropriate. $\mathrm{P}<0.05$ was considered significant.

\section{RESULTS}

\section{Characteristics of the Subjects' Groups}

Clinical parameters of patients and NCs are shown in Table $\mathbf{1 .}$ WBC values were significantly higher in patients than in NC. Platelets were significantly higher in NC than in patients, although they were within the normal range. No significant differences were observed in all other parameters.

\section{The Levels of Cell-Free IgG-Hexamers in Patients and NC}

Protein G columns and 1,000 kDa filtration columns were used for separation of IgG-hexamers, and the percent of IgG-hexamers were calculated. The percent of IgG hexamers in the NC samples was $9.8 \pm 5 \%$ of the total IgG while significantly higher percentage was observed in patients with and without chronic complement activation [according to the detection of Ig-C5a (10)], showing $19.53 \pm 3 \%$ and $20.26 \pm 4 \%$, respectively (Figure 1A). The presence of IgG-hexamers was also verified by another separation method (The Melon Gel Kit), which does not include an elution step of the IgG by a low $\mathrm{pH}$. The percentage of IgG-hexamers obtained by the Melon Gel Kit and the Protein G columns showed significant correlation (Supplementary Data Sheet 1). The identity of the separated IgG-hexamers was verified by Western blot using anti-human IgG antibodies (Figure 1B). 
TABLE 1 | Characteristics of the subjects' groups.

\begin{tabular}{|c|c|c|}
\hline Clinical Parameters (normal range) & CLL Patients & Normal Controls \\
\hline$n$ & 30 & 12 \\
\hline Gender (male/female) & $14 / 16$ & $5 / 7$ \\
\hline Age (years) & $69.4 \pm 10.9$ & $59.7 \pm 10.1$ \\
\hline Serum C3 mg/dl (82-158) & $109.7 \pm 23.9$ & $125.4 \pm 21.5$ \\
\hline Serum C4 mg/dl (15-35) & $28.2 \pm 8.8$ & $29.3 \pm 2.3$ \\
\hline Cholesterol mg/dl (<200) & $158.1 \pm 35.2$ & $203.6 \pm 80.9$ \\
\hline Triglycerides mg/dl (<150) & $144.3 \pm 78.5$ & $177.1 \pm 72.9$ \\
\hline $\mathrm{HDL} \mathrm{mg/dl}(>40)$ & $37.8 \pm 13.6$ & $43.7 \pm 9.6$ \\
\hline LDL mg/dl (<100) & $91.3 \pm 38.7$ & $112 \pm 52.4$ \\
\hline Non-HDL chol. mg/dl (<130) & $120.1 \pm 31.2$ & $160.1 \pm 80.5$ \\
\hline 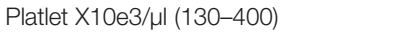 & $164.17 \pm 75.9^{*}$ & $272.5 \pm 98.3$ \\
\hline WBC X10e3/Hl (4-10) & $33.3 \pm 69.3^{\star}$ & $7.4 \pm 2.8$ \\
\hline HGB g/dl (13-18) & $13 \pm 2.01$ & $13.6 \pm 1.1$ \\
\hline 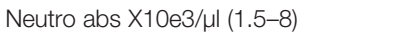 & $4.6 \pm 2.1$ & $4.7 \pm 2.4$ \\
\hline
\end{tabular}

All values are given as mean \pm SEM.

*indicates significant $p$ value $(p<0.05)$.

A

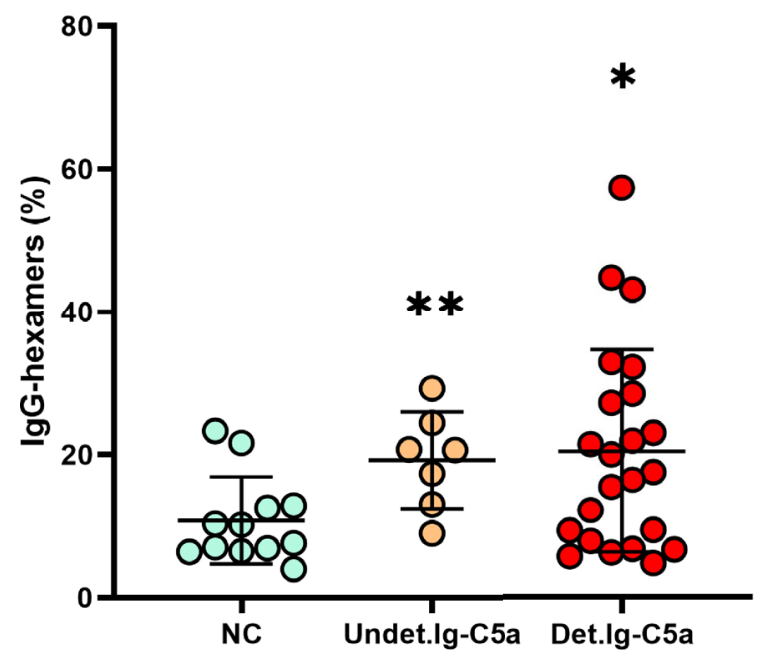

B

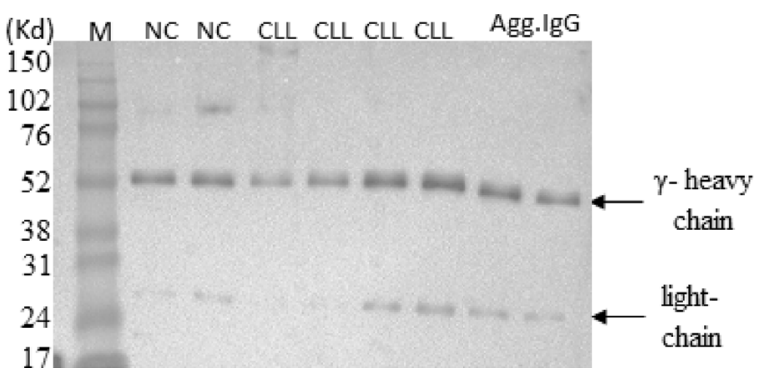

FIGURE 1 | Cell free lgG-hexamers patients and NC subjects. IgG-hexamers were purified from sera/plasma of CLL patients and NC subjects. (A) The percentage of lgG-hexamers were calculated. Detectable lg-C5a (Det.lg-C5a), $\mathrm{n}=23$, undetectable Ig-C5a (Undet.lg-C5a) $\mathrm{n}=7, \mathrm{NC} n=12$. *, ** indicates significant $p$ values ( $p<0.04,0.004$, respectively) vs NC. (B) The samples were studied by Western blot using anti-IgG antibodies (representative results), equal protein amounts (10 $\mu$ g) were loaded in each lane. In vitro aggregated commercial lgG (Agg.lgG) was separated in parallel as a control. The heavy and light chains of the lgG are indicated by arrows.

\section{IgG-Hexamers Contribute to the Chronic CP Activation in CLL Patients Serum}

To assess the CP activation capacity of the IgG-hexamers, the CP was activated in normal serum by addition of the IgG-hexamers' preparations, and activity was assessed by the levels of the sC5b-9 produced (Figure 2). IgG-hexamers from patients with detectable Ig-C5a activated complement to a higher extent than those from NC plasma. IgG-hexamers from patients with undetectable Ig-C5a did not show similar results (Figure 2A). Non-IgG proteins (proteins that did not bind to the protein G columns) were able to activate complement in C1q-depleted serum (Figure 2B) suggesting activation via the AP. The data obtained using factor B depleted serum (Figure 2C) support AP activation. Non-hexameric (monomeric) IgG samples from patients or NC did not induce complement activation.

\section{Cell-Bound IgG-Hexamers Are Detected on B Cells}

Fresh blood samples from NC and CLL patients were stained with fluorescent antibodies against CD45, CD19 and C1 and tested in a flow cytometer in order to assess IgG-hexamers that are present on cells' surfaces. The results showed that the anti-C1 antibody stained WBC $\left(\mathrm{CD}_{4} 5^{+}\right)$and particularly B cells $\left(\mathrm{CD} 19^{+}\right)$. In $\mathrm{NC}$ the $\mathrm{C} 1+\mathrm{CD} 19+$ staining was $20 \pm 10 \%$ and $65 \pm 8 \%$ in patients, when gated on WBC (Figure 3A, representative results and Figure 3C). In order to overcome the increase in B cell population in the patients, analysis was performed again after gating on lymphocytes. The percent of $\mathrm{C} 1+\mathrm{CD} 19+$ cells was significantly higher in patients, showing $95 \pm 3 \%$, compared to only $60 \pm 20 \%$ in NC (Figure 3B, representative results and Figure 3D). The results were negatively correlated with the levels of cell-free IgGhexamers $(\mathrm{p}<0.03)$. This observation suggests that cell-free IgGhexamers are in equilibrium with the cell-bound hexamers found on B-cell surfaces.

\section{Identity of the Antigen That Is Causing the Hexamerization}

Ten $\mu \mathrm{g}$ of the IgG-hexamer samples were used for SDS-PAGE and silver staining. The results showed additional proteins besides the heavy and light chains of the IgG (Figure 4A). These were excised from the gel and subjected to mass spectrometry. After elimination of all the IgG-related sequences, low molecular mass peptides, sequences with a total number of identified peptide sequences (peptide spectrum matches-\#PSMs) $<30$, and sequences with coverage $<25$, the results indicated six proteins suspected as the antigens that may cause the IgG-hexamerization (Figure 4B). One of these candidates was found to be the A2M. The raw sequencing data of the A2M identified peptides can be accessed in the Dryad database under the accession number https://doi.org/10.5061/ dryad.tmpg $4 \mathrm{f} 4 \mathrm{xg}$. A2M was further studied by Western blot using anti-A2M antibody to verify the presence of A2M in the IgGhexamer preparations. The Western blot results indicated A2M presence in IgG-hexamer samples of CLL patients (Figure 5) and very low or no $\mathrm{A} 2 \mathrm{M}$ signal in $\mathrm{NC}$ samples, indicating its participation in the hexamer structure. A2M showed a MW of $\sim 360 \mathrm{kDa}$, similar to that of the purified commercial A2M that was used as a positive control (Figure 5). 
A

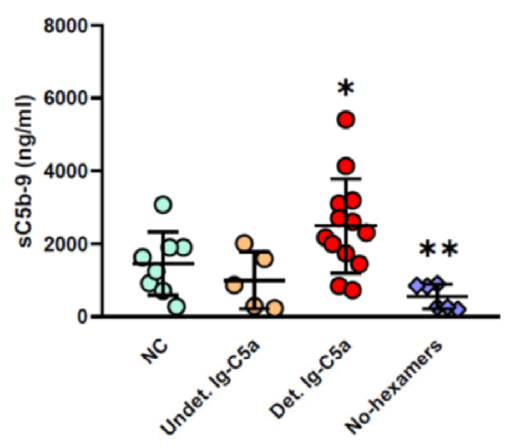

B

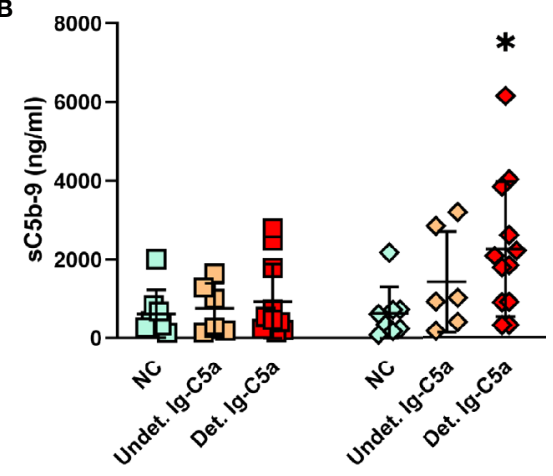

C

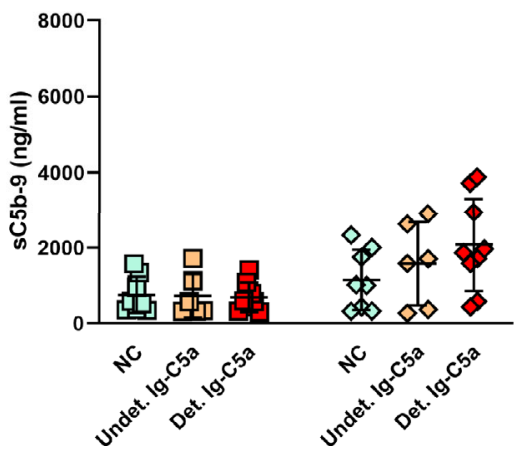

FIGURE 2 | Activation of the complement system by lgG-hexamers. Complement activity was measured in normal serum after incubation with IgG-hexamers from $\mathrm{NC}$ and patients (A). Serum samples incubated with buffer were used as a negative control. Proteins that did not bind to the protein $\mathrm{G}$ columns (non-lgG proteins, $\diamond$ ), and non-hexameric lgG (monomeric lgG, $\square$ ) were used for complement activation in C1q depleted serum (B) and factor B depleted serum (C). Activation was followed by the levels of sC5b-9. Detectable Ig-C5a (Det.lg-C5a) $n=12$, Undetectable Ig-C5a (Undet.lg-C5a) $n=6$, NC $n=8$. *, ** indicates significant $p$ values ( $\mathrm{p}<0.05,0.005$, respectively) compared to NC and No-hexamers.

The association of the A2M-IgG-hexamers with B lymphocytes was studied by flow cytometry. Fresh blood samples from NC and CLL patients were stained with fluorescent antibodies to A2M, C1 and two A2M receptors, the CD91 (data not shown) and GRP78. High A2M+GRP78+ staining in $\mathrm{C} 1+\mathrm{CD} 19+$ cells supports the presence of A2M-IgG-hexamers on B-cells in addition to the cellfree form (Figure 6). The data suggest that the A2M-IgGhexamers are attached to B-cells by binding the GRP78 (Figure 6).

\section{Monomeric IgG Form Hexamers in-Vitro After Incubation with A2M in a Cell-Free Environment}

Our working assumption was that IgG molecules which bind $\mathrm{A} 2 \mathrm{M}$ are those that form hexamers/aggregates. We assessed this assumption by incubation of total serum monomeric IgG with purified commercial A2M, followed by separation and measurement of the generated hexamers. The results shown in Figure 7 indicate a significant increase in IgG-hexamer generation after incubation of A2M with monomeric IgG from patients with detectable Ig-C5a. Hexamer generation without addition of $\mathrm{A} 2 \mathrm{M}$ was minimal, only $4.9 \%$ to $7.8 \%$ and without significant differences between the subject groups. A nonsignificant increase in IgG-hexamers was found in the NC group, and no increase was observed in patients with undetectable Ig-C5a (Figure 7). Also, no hexamerization was observed after incubation of IgG with a different protein, HSA. The results indicate the presence of anti-A2M antibodies in sera of patients with detectable Ig-C5a (chronic complement activation) and support the potential formation of hexamers in a cell-free environment, i.e. in the plasma.

\section{DISCUSSION}

Our study focused on characterization of the IgG-hexamers that are related to chronic CP activation in CLL. IgG-hexamers normally bind $\mathrm{C} 1$, the first component of the $\mathrm{CP}$, and activate a cascade of complement proteins until C5b-9 is formed. The high level of these cell-free hexamers in patients has a key role in chronic CP activation, leading to the "weariness" of this pathway as well as to an increase in the formation of Ig-C5a complex and in the levels of other complement activation markers (17). IgGhexamers are mainly formed after antigen binding that leads to non-covalent Fc-Fc interactions (23). We assumed that in CLL patients a particular antigen (or antigens) causes increased formation of IgG-hexamers, as shown by their high percentages in the patients' plasma. In this study, the antigen causing hexamerization was found to be A2M. 

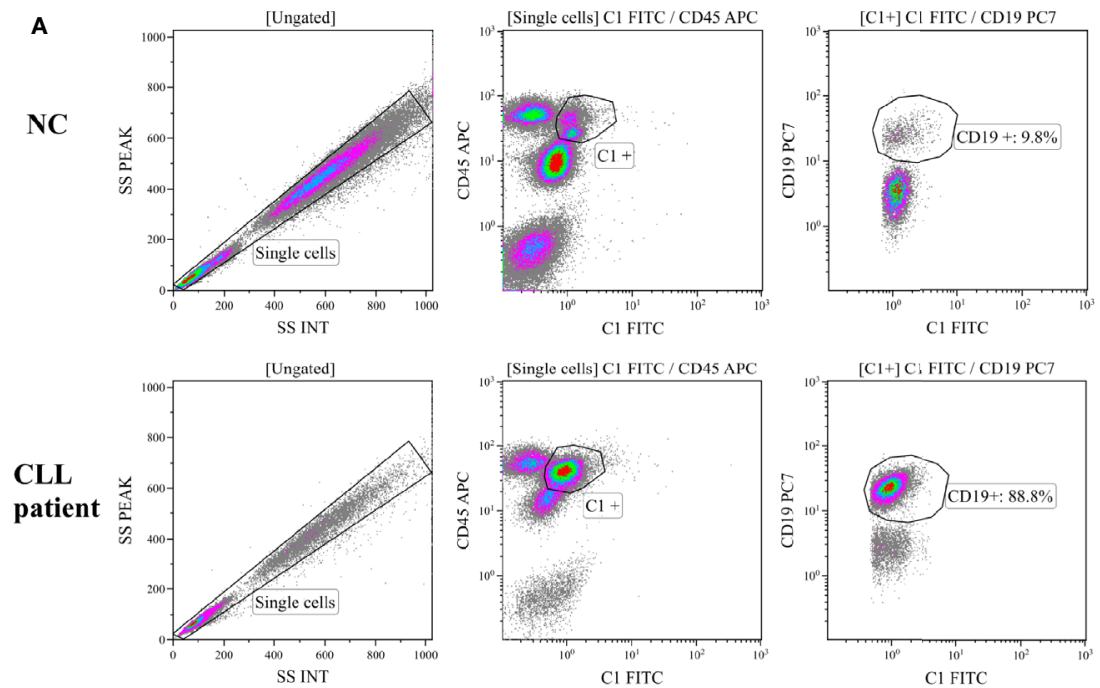

B
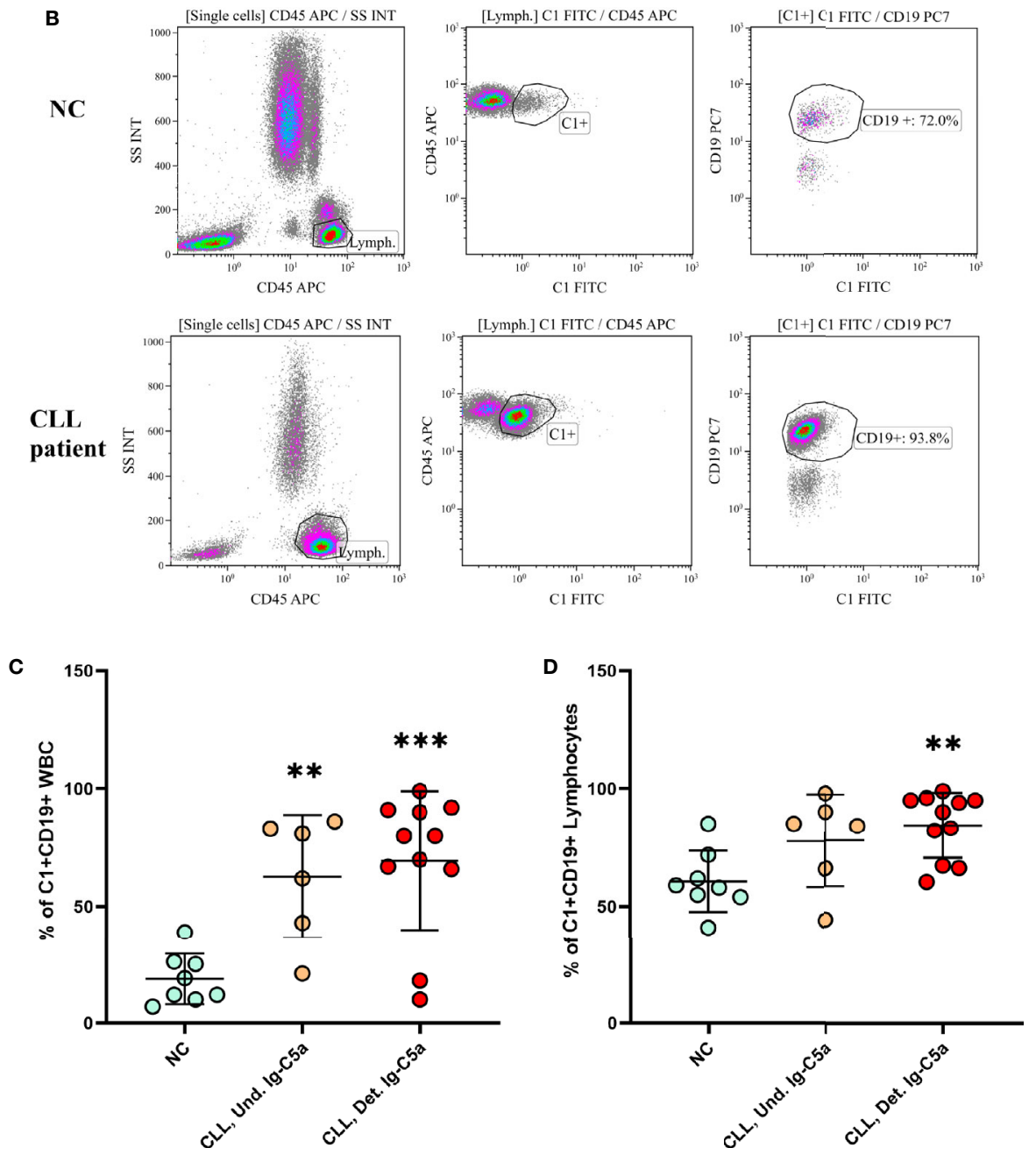

FIGURE 3 | IgG-hexamers on B cell surface. Blood samples from CLL patients and NC were stained with fluorescent antibodies against CD45, CD19 and C1, and tested in a flow cytometer. representative results are shown $(\mathbf{A}, \mathbf{B})$. The results were gated on WBC $(\mathbf{A}, \mathbf{C})$ or on lymphocytes $(\mathbf{B}, \mathbf{D})$. Detectable lg-C5a $n=11$; Undetectable Ig-C5a $n=6$; NC $n=8$. ${ }^{\star *},{ }^{\star \star \star}$ indicate significant $p$ values $(p<0.01,0.001$, respectively) compared to NC. 

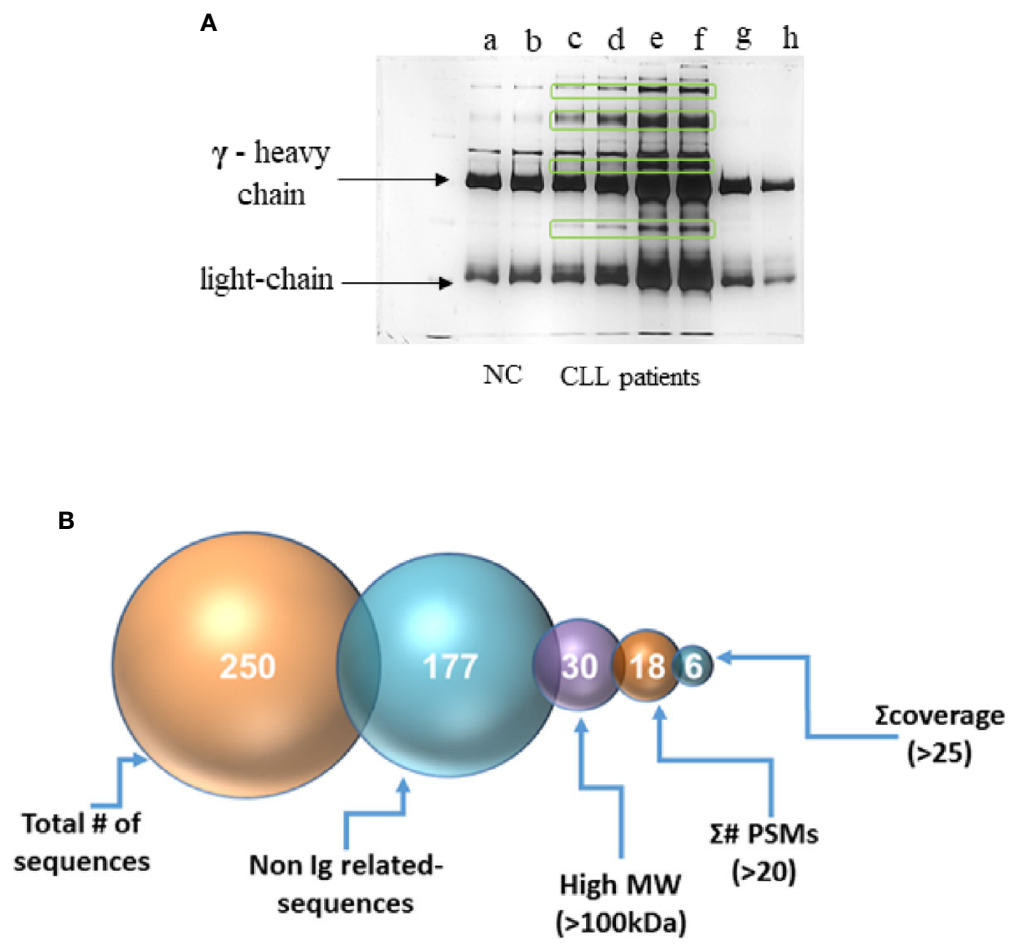

FIGURE 4 | Separation of the IgG-hexamer samples. (A) Samples of NC (a, b), CLL patients (c-f) and commercial lgG (g, h) were separated and silver stained. Heavy $(\gamma)$ and light chain are indicated by arrows. Additional proteins besides heavy and light chains are marked in frames. (B) The process of selection of the resulting sequence data included elimination of all the lgG-related sequences, low molecular mass peptides, sequences with a total number of identified peptide sequences (peptide spectrum matches-\#PSMs)<30, and sequences with coverage<25.

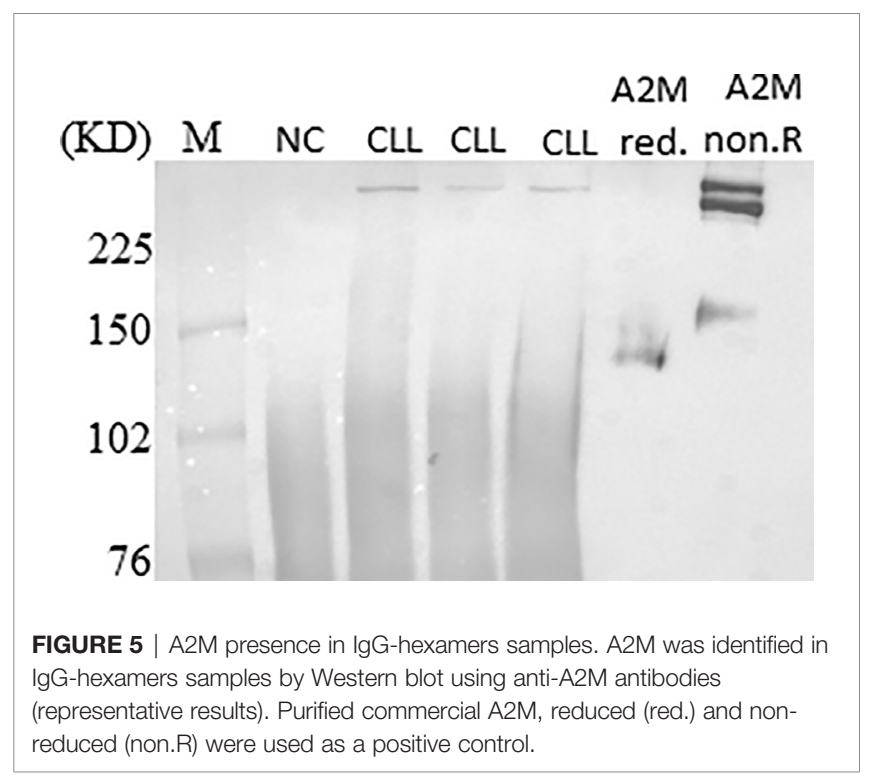

The IgG-antigen aggregates (immune complexes) exist on the surface of malignant B cells and also as a detached, cell-free, form. The percentage of both cell-free and cell-bound IgG-hexamers is significantly higher in patients than in NC. The increased ability of these hexamers/aggregates to activate the $\mathrm{CP}$ supports their potential role in the chronic activation of the CP in CLL.
It is important to mention that in this study we show that natural IgG aggregates exist in complex with A2M, but we did not demonstrate the hexameric rings. Thus, the complexes formed between IgG and A2M may potentially involve Fab-mediated clustering of dimeric $\mathrm{A} 2 \mathrm{M}$, in addition to the known $\mathrm{Fc}: \mathrm{Fc}$ interactions. In this case, large aggregated networks may be formed, and these aggregates may include complexes including more than six IgG molecules (17). These aggregates are very potent in complement activation and in depleting the complement system in CLL patients.

During normal physiological conditions, AP dominates activation of the complement system in plasma due to the spontaneous hydrolysis of complement component C3 (24). This hydrolysis can be hastened through some biological interfaces that include lipid complexes (8). Moreover, non-IgG proteins (from CLL patients with chronic complement activation) may also activate the AP, as suggested in this study by the data obtained using $\mathrm{Clq}$ depleted serum.

A previous study showed decreased expression of CD19 molecules on B-cells from CLL patients compared to normal B-cells (25). This information supports the significance of our findings on the differences in $\mathrm{C} 1+\mathrm{CD} 19+$ cell populations between patients and NCs and suggests that our findings are truly due to a higher presence of IgG-hexamers on B cell surface in the patients, rather than due to increased expression of CD19.

One or several of the additional non-IgG proteins that were found in the IgG-hexamer preparations was the antigen/s 

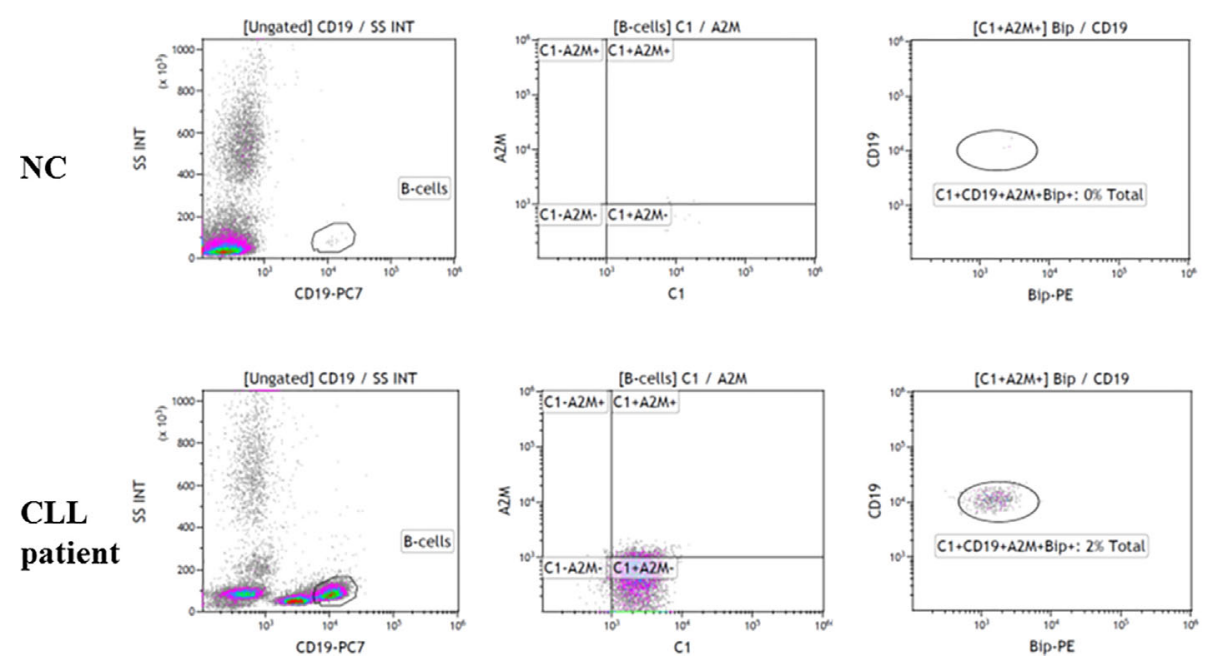

FIGURE 6 | Association of the A2M-lgG-hexamers with B lymphocytes. Blood samples from CLL patients and NC were stained with fluorescent antibodies against CD45, CD19, C1, A2M and GRP78 (Bip) and tested in a flow cytometer. Representative results are shown.

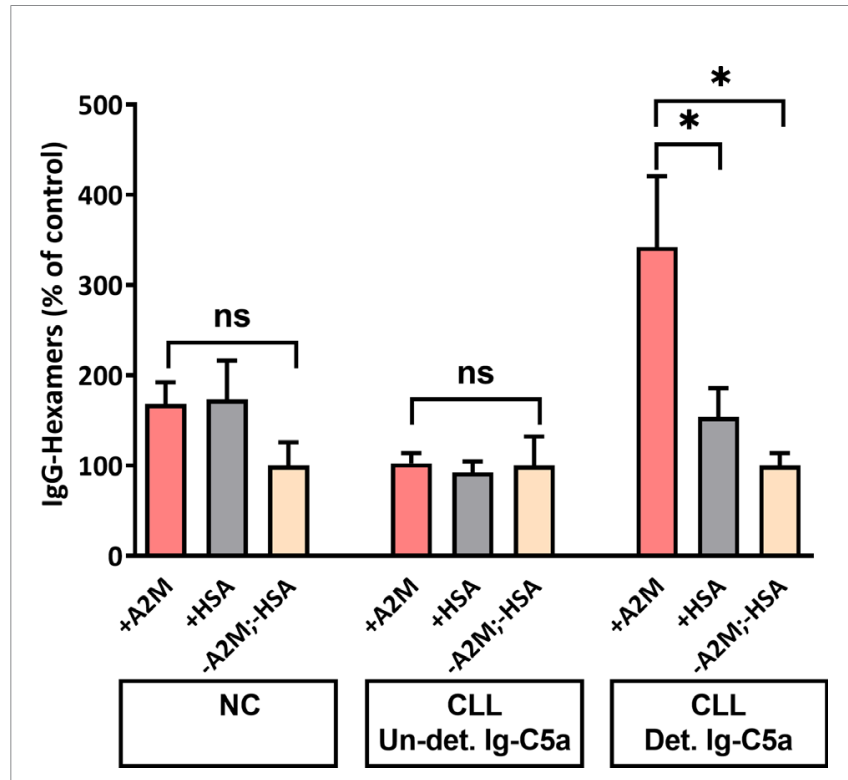

FIGURE 7 | IgG-hexamer formation after in-vitro incubation of monomeric IgG with A2M. Monomeric IgG were incubated with purified commercial A2M, HSA or PBS and the generated IgG-hexamers were separated. IgG were quantified by ELISA and the percent of IgG-hexamers was calculated.

*, ns indicate significant $(<0.05)$ and non-significant $p$ values, respectively.

causing the hexamerization. Mass spectrometry (protein sequencing) analysis indicated that $\mathrm{A} 2 \mathrm{M}$ could be the antigen. A2M is formed by the assembly of four $180-\mathrm{kDa}$ subunits into two disulfide-linked dimers, which noncovalently associate to complete the tetrameric structure of A2M (26). However, several studies showed that A2M is present in the circulation in either a dimeric or tetrameric form $(27,28)$. A2M can bind through the CD91 receptor to $\mathrm{T}$ cells
(20), and one study showed that A2M could also be expressed on B lymphocytes (29). Another receptor for A2M is GRP78, a member of the HSP70 gene family (30), whose levels are elevated in hypoxia and starvation conditions, allowing it to function as a shield for solid tumors in these cases (31). Studies have shown that GRP78 is mainly expressed on B cells compared to $\mathrm{T}$ cells and its levels are significantly higher in patients with CLL than in NC (32).

Both the Western blot and flow cytometry analyses confirmed that A2M was the antigen causing IgG hexamerization. The data suggest that A2M-IgG-hexamers do not necessarily form on the surface of B cells as they can also form in plasma before binding to the cells via A2M receptors. Antibodies that react against A2M may be part of a more general autoimmune phenomenon which is established occasionally in CLL patients $(33,34)$.

In hematologic malignancies, $\mathrm{C}$ activation can have both protective properties as well as tumor growth promoting effects, both direct and indirect (35). For example, hematological tumor cells show enhanced expression and surface binding of $\mathrm{C}$ regulators such as Factor $\mathrm{H}$, Factor $\mathrm{H}$ like protein 1 (FHL-1), Factor $\mathrm{H}$ related protein 1 (CFHR1), FHR-4, FHR5, and C4b binding protein $(\mathrm{C} 4 \mathrm{BP})$. These $\mathrm{C}$ regulators further display the cofactor activity, which function together with factor I to block $\mathrm{C}$ activation at the level of C3 convertase, and lead to C evasion (35). Similarly to these soluble regulators, the membrane-bound $\mathrm{C}$ inhibitors CD46, CD55 and CD59 are up-regulated in various primary tumors and tumor lines to evade the C attack (35). Also, the soluble form of the $\mathrm{C}$ receptor 1 (CR1), expressed on most leukocytes membranes, is shed into the plasma, where it functions as a powerful $\mathrm{C}$ inhibitor. This represents another possible $\mathrm{C}$ evasion strategy utilized by leukemia cells (35).

Although our findings regarding the A2M-IgG-hexamers are novel, the factors that control the hexamerization process and the sequence of their formation in CLL plasma and on B-cells 
have not yet been revealed and need further clarification. For the benefit of the patients, the results may be useful for improvement of current immunotherapy treatments, for example if A2M-IgGhexamerization and the resulting chronic $\mathrm{CP}$ activation can be inhibited, and thus help the complement system attain maximal capacity. Alternatively, measurements of this protein or other complement activity markers can help physicians to identify patients who may be less responsive to immunotherapy. For these patients, other therapeutic options should be offered.

\section{DATA AVAILABILITY STATEMENT}

The raw sequencing data of the A2M identified peptides can be accessed in the Dryad database under the accession number https://doi.org/10.5061/dryad.tmpg4f4xg.

\section{ETHICS STATEMENT}

The studies involving human participants were reviewed and approved by Institutional Review Board of Galilee Medical Center, Nahariya, Israel. The patients/participants provided their written informed consent to participate in this study.

\section{AUTHOR CONTRIBUTIONS}

$\mathrm{NN}$ : data curation, methodology. RM: conceptualization, data curation, data analysis, methodology, project administration,

\section{REFERENCES}

1. Mohr A, Renaudineau Y, Bagacean C, Pers JO, Jamin C, Bordron A. Regulatory B lymphocyte functions should be considered in chronic lymphocytic leukemia. Oncoimmunology (2016) 5:e1132977. doi: 10.1080/ 2162402X.2015.1132977

2. Marabelle A, Tselikas L, de Baere T, Houot R. Intratumoral immunotherapy: using the tumor as the remedy. Ann Oncol (2017) 28:33-43. doi: 10.1093/ annonc/mdx683

3. Chien WW, Niogret C, Jugé R, Lionnard L, Cornut-Thibaut A, Kucharczak J, et al. Unexpected cross-reactivity of anti-cathepsin B antibodies leads to uncertainties regarding the mechanism of action of anti-CD20 monoclonal antibody GA101. Leuk Res (2017) 55:41-8. doi: 10.1016/ j.leukres.2017.01.010

4. Weiner LM, Surana R, Wang S. Monoclonal antibodies: versatile platforms for cancer immunotherapy. Nat Rev Immunol (2010) 10:317-27. doi: 10.1038/ nri2744

5. Jaglowski SM, Alinari L, Lapalombella R, Muthusamy N, Byrd JC. Review article The clinical application of monoclonal antibodies in chronic lymphocytic leukemia. Blood (2010) 116:3705-14. doi: 10.1182/blood-201004-001230

6. Dunkelberger JR, Song WC. Complement and its role in innate and adaptive immune responses. Cell Res (2010) 20:34-50. doi: 10.1038/cr.2009.139

7. Pio R, Corrales L, Lambris JD. The role of complement in tumor growth.. Adv Exp Med Biol (2014) 772:229-62. doi: 10.1007/978-1-4614-5915-6_11

8. Merle NS, Church SE, Fremeaux-Bacchi V, Roumenina LT. Complement System Part I - Molecular Mechanisms of Activation and Regulation. Front Immunol (2015) 6:262. doi: 10.3389/fimmu.2015.00262 supervision, validation, writing - original draft, writing - review and editing. MB: project administration, supervision. JC: data curation, data analysis, methodology. TT: conceptualization, resources. AA: resources. LS: resources. AL: resources. MS: methodology. GS: resources. ES: methodology. AB: supervision, resources, project administration, methodology, funding, conceptualization, review and editing. All authors contributed to the article and approved the submitted version.

\section{FUNDING}

The study was funded by the Health Corporation of Galilee Medical Center.

\section{ACKNOWLEDGMENTS}

The authors thank Mrs. Tobie Kuritsky and Prof. Chaim Putterman for their help in editing and proofreading of the manuscript, and Mr. Basem Hijazi, a professional statistician, for his help with the statistical analysis.

\section{SUPPLEMENTARY MATERIAL}

The Supplementary Material for this article can be found online at https://www.frontiersin.org/articles/10.3389/fimmu.2020. 603569/full\#supplementary-material

9. Schlesinger M, Broman I, Lugassy G. The complement system is defective in chronic lymphatic leukemia patients and in their healthy relatives. Leukemia (1996) 10:1509-13

10. Michelis R, Tadmor T, Barhoum M, Shehadeh M, Shvidel L, Aviv A, et al. A C5a-Immunoglobulin complex in chronic lymphocytic leukemia patients is associated with decreased complement activity. PloS One (2019) 14:1-17. doi: 10.1371/journal.pone.0209024

11. Middleton O, Cosimo E, Dobbin E, McCaig AM, Clarke C, Brant AM, et al. Complement deficiencies limit CD20 monoclonal antibody treatment efficacy in CLL. Leukemia (2015) 29:107-14. doi: 10.1038/leu.2014.146

12. Arlaud GJ, Gaboriaud C, Thielens NM, Budayova-Spano M, Rossi V, Fontecilla-Camps JC. Structural biology of the C1 complex of complement unveils the mechanisms of its activation and proteolytic activity. Mol Immunol (2002) 39:383-94. doi: 10.1016/s0161-5890(02)00143-8

13. Mortensen SA, Sander B, Jensen RK, Pedersen JS, Golas MM, Jensenius JC, et al. Structure and activation of $\mathrm{C} 1$, the complex initiating the classical pathway of the complement cascade. Proc Natl Acad Sci U S A (2017) 114:986-91. doi: 10.1073/pnas.1616998114

14. Hansch RGOTGM. chapter 1.2.1, Classical Pathway of Activation. In: Edition $R$. The Complement System. 2nd Revise. Heidelberg Germany: Springer-Verlag Berlin Heidelberg New York (1998). p. 68-85.

15. Cook EM, Lindorfer MA, van der Horst H, Oostindie S, Beurskens FJ, Schuurman J, et al. Antibodies That Efficiently Form Hexamers upon Antigen Binding Can Induce Complement-Dependent Cytotoxicity under Complement-Limiting Conditions. J Immunol (2016) 197:1762-75. doi: 10.4049/jimmunol.1600648

16. Diebolder CA, Beurskens FJ, de Jong RN, Koning RI, Strumane K, Lindorfer MA, et al. Complement is activated by IgG hexamers assembled at the cell surface. Science (2014) 343:1260-3. doi: 10.1126/science.1248943 
17. Michelis R, Tadmor T, Aviv A, Stemer G, Majdob R, Shvidel L, et al. Cell-free IgG-aggregates in plasma of patients with chronic lymphocytic leukemia cause chronic activation of the classical complement pathway. PloS One (2020) 15: e0230033. doi: 10.1371/journal.pone.0230033 Published 2020 Mar 9.

18. Mollnes TE, Garred P, Bergseth G. Effect of time, temperature and anticoagulants on in vitro complement activation: consequences for collection and preservation of samples to be examined for complement activation. Clin Exp Immunol (1988) 73:484-8.

19. Blom AM, Österborg A, Mollnes TE, Okroj M. Antibodies reactive to cleaved sites in complement proteins enable highly specific measurement of soluble markers of complement activation. Mol Immunol (2015) 66:164-70. doi: 10.1016/j.molimm.2015.02.029

20. Binder RJ, Karimeddini D, Srivastava PK. Adjuvanticity of alpha 2macroglobulin, an independent ligand for the heat shock protein receptor CD91. J Immunol (2001) 166:4968-72. doi: 10.4049/jimmunol.166.8.4968

21. Zhang LH, Zhang X. Roles of GRP78 in physiology and cancer. J Cell Biochem (2010) 110:1299-305. doi: 10.1002/jcb.22679

22. Biltoft D, Gram JB, Larsen A, Münster AB, Sidelmann JJ, Skjoedt K, et al. Fast form alpha-2-macroglobulin - A marker for protease activation in plasma exposed to artificial surfaces. Clin Biochem (2017) 50:1203-8. doi: 10.1016/ j.clinbiochem.2017.09.002

23. Strasser J, de Jong RN, Beurskens FJ, Wang G, Heck AJR, Schuurman J, et al. Unraveling the Macromolecular Pathways of IgG Oligomerization and Complement Activation on Antigenic Surfaces. Nano Lett (2019) 19:478796. doi: 10.1021/acs.nanolett.9b02220

24. Zhou X, Hu W, Qin X. The role of complement in the mechanism of action of rituximab for B-cell lymphoma: implications for therapy. Oncologist (2008) 13:954-66. doi: 10.1634/theoncologist.2008-0089

25. Ginaldi L, De Martinis M, Matutes E, Farahat N, Morilla R, Catovsky D. Levels of expression of CD19 and CD20 in chronic B cell leukaemias. J Clin Pathol (1998) 51:364-9. doi: 10.1136/jcp.51.5.364

26. Cater JH, Wilson MR, Wyatt AR. Alpha-2-Macroglobulin, a HypochloriteRegulated Chaperone and Immune System Modulator. Oxid Med Cell Longev (2019) 2019:5410657. doi: 10.1155/2019/5410657

27. Garcia-Ferrer I, Marrero A, Gomis-Rüth FX, Goulas T. $\alpha 2$-Macroglobulins: Structure and Function. Subcell Biochem (2017) 83:149-83. doi: 10.1007/9783-319-46503-6_6

28. Pitekova B, Kupcova V, Uhlikova E, Mojto V, Turecky L. Alpha-2macroglobulin and hyaluronic acid as fibromarkers in patients with chronic hepatitis C. Bratisl Lek Listy (2017) 118:658-61. doi: 10.4149/ BLL_2017_125

29. James K, Tunstall AM, Parker AC, McCormick JN. The association of alpha2macroglobulin with lymphocyte membranes in chronic lymphocytic leukaemia and other disorders. Clin Exp Immunol (1975) 19:237-49.

30. Ni M, Zhang Y, Lee AS. Beyond the endoplasmic reticulum: atypical GRP78 in cell viability, signalling and therapeutic targeting. Biochem J (2011) 434:181-8. doi: 10.1042/BJ20101569

31. Ijabi R, Roozehdar P, Afrisham R, Moradi-Sardareh H, Kaviani S, Ijabi J, et al. Association of GRP78, HIF-1 $\alpha$ and BAG3 Expression with the Severity of Chronic Lymphocytic Leukemia. Anticancer Agents Med Chem (2020) 20:429-36. doi: 10.2174/1871520619666191211101357

32. Huergo-Zapico L, Gonzalez-Rodriguez AP, Contesti J, Gonzalez E, LópezSoto A, Fernandez-Guizan A, et al. Expression of ERp5 and GRP78 on the membrane of chronic lymphocytic leukemia cells: association with soluble MICA shedding. Cancer Immunol Immunother (2012) 61:1201-10. doi: 10.1007/s00262-011-1195-Z

33. Vitale C, Montalbano MC, Salvetti C, Boccellato E, Griggio V, Boccadoro M, et al. Autoimmune Complications in Chronic Lymphocytic Leukemia in the Era of Targeted Drugs. Cancers (Basel) (2020) 12:282. doi: 10.3390/cancers 12020282

34. Forconi F, Moss P. Perturbation of the normal immune system in patients with CLL. Blood (2015) 126:573-81. doi: 10.1182/blood-2015-03-567388

35. Luo S, Wang M, Wang H, Hu D, Zipfel PF, Hu Y. How Does Complement Affect Hematological Malignancies: From Basic Mechanisms to Clinical Application. Front Immunol (2020) 11:593610. doi: 10.3389/fimmu.2020. 593610

Conflict of Interest: The authors declare that the research was conducted in the absence of any commercial or financial relationships that could be construed as a potential conflict of interest.

Copyright (c) 2021 Naseraldeen, Michelis, Barhoum, Chezar, Tadmor, Aviv, Shvidel, Litmanovich, Shehadeh, Stemer, Shaoul and Braester. This is an open-access article distributed under the terms of the Creative Commons Attribution License (CC BY). The use, distribution or reproduction in other forums is permitted, provided the original author(s) and the copyright owner(s) are credited and that the original publication in this journal is cited, in accordance with accepted academic practice. No use, distribution or reproduction is permitted which does not comply with these terms. 